\title{
Beam-Sample Interactions During Liquid Cell Electron Microscopy
}

\author{
Joseph M. Grogan ${ }^{1}$, Frances M. Ross ${ }^{2}$, and Haim H. Bau ${ }^{1}$ \\ 1. Department of Mechanical Engineering and Applied Mechanics, University of Pennsylvania, \\ Philadelphia, PA 19104, USA. \\ 2. IBM T. J. Watson Research Center, Yorktown Heights, NY 10598, USA.
}

Liquid cell electron microscopy is a powerful in situ technique that uses the transmission (TEM) or scanning transmission (STEM) electron microscope to image micro- and nanoscale phenomena in liquid media [1,2]. The technique has been used to image phenomena such as nucleation and growth of nanoparticles; electrochemical processes; motion, aggregation, and assembly of suspended nanoparticles; boiling; macromolecular conformations; and biological processes in cells. For better or worse, the electron beam interacts with the sample during imaging and may affect the phenomena under investigation. In fact, many in situ crystallization and growth studies rely on the beam to drive the process. The interaction between electrons and the liquid medium, a critically important issue, is only now starting to attract detailed attention from the liquid cell microscopy community. A clear understanding of this issue is essential to account for, suppress, or exploit beam effects.

Although electron beam interactions with solid matter have been studied in detail [3], the situation in liquids differs because of the high mobility of species in the sample. However, the interaction of ionizing radiation with fluid media has been studied by radiation chemists for decades, and is of key importance in nuclear science, medical imaging and therapeutics, food preservation, and other manufacturing processes. Much of the knowledge acquired in these fields is applicable to liquid cell (S)TEM, and conversely, the liquid cell provides a unique tool to study radiation effects. However, it is important to note that the radiation dose-rates in electron microscopy far exceed the levels encountered in most other applications of radiation chemistry.

Here we describe the interactions of high energy electrons with water, finding that radiolysis plays an important role and heating is typically insignificant. In order to develop a model for beam effects, we examine beam-induced bubble formation (Figure 1). We hypothesize that $\mathrm{H}_{2}$ produced by water radiolysis is the main cause for bubble formation during electron imaging. We then develop a simplified reaction-diffusion model for beam-induced production of $\mathrm{H}_{2}$ and compare the theoretical predictions with experimental observations of the rate of bubble formation and growth. An example is shown in Figure 2, where the $\mathrm{H}_{2}$ concentration is calculated in the region around two nearby bubbles. The model predicts that within seconds to minutes of imaging with only moderate beam current, the concentration of $\mathrm{H}_{2}$ can exceed its saturation concentration, making bubble nucleation possible. Our study yields two key findings. First: radiolysis byproducts formed by the beam quickly reach chemical equilibrium, with a steady-state concentration that is dose-rate dependent. And second: bubble growth is very sensitive and responds superlinearly to beam current.

Based on the results from this study, we suggest that further systematic examination of radiolysis effects specific to the conditions of the electron microscope is of key importance for obtaining quantitative data from liquid cell microscopy. Highly reactive species are continuously produced during (S)TEM observation and the invisible beam effects cannot be dismissed just because there are no bubbles. The byproducts of water radiolysis (primarily: $\mathrm{e}_{\mathrm{h}}, \mathrm{H}_{3} \mathrm{O}^{+}, \mathrm{H}, \mathrm{OH}, \mathrm{H}_{2}$, and $\mathrm{H}_{2} \mathrm{O}_{2}$ ) are strong oxidizing and 
reducing agents that are likely to be responsible for a variety of phenomena observed in liquid cell electron microscopy [4].

\section{References:}

[1] N. de Jonge and F. M. Ross, Nature Nanotechnology, volume 6, no. 11 (2011), p. 695-704.

[2] J. M. Grogan, N. M. Schneider, F. M. Ross, and H. H. Bau, Journal of the Indian Institute of Science, volume 92, no. 2 (2012), p. 295-308.

[3] R. F. Egerton, P. Li, and M. Malac, Micron, volume 35 (2004), p. 339-409.

[4] The authors acknowledge funding from the National Science Foundation, grants 1129722 and 1066573. Electron microscopy was performed at the Penn Regional Nanotechnology Facility and the IBM T. J. Watson Research Center with the valuable assistance of Mr. Peter Szczesniak of UPenn and Dr. Mark C. Reuter and Mr. Arthur Ellis of IBM. Dr. Jay A. LaVerne (Notre Dame Radiation Laboratory), Dr. Ray Egerton (University of Alberta, Physics), and Dr. Brian Edwards (UPenn, ESE) provided useful discussions.
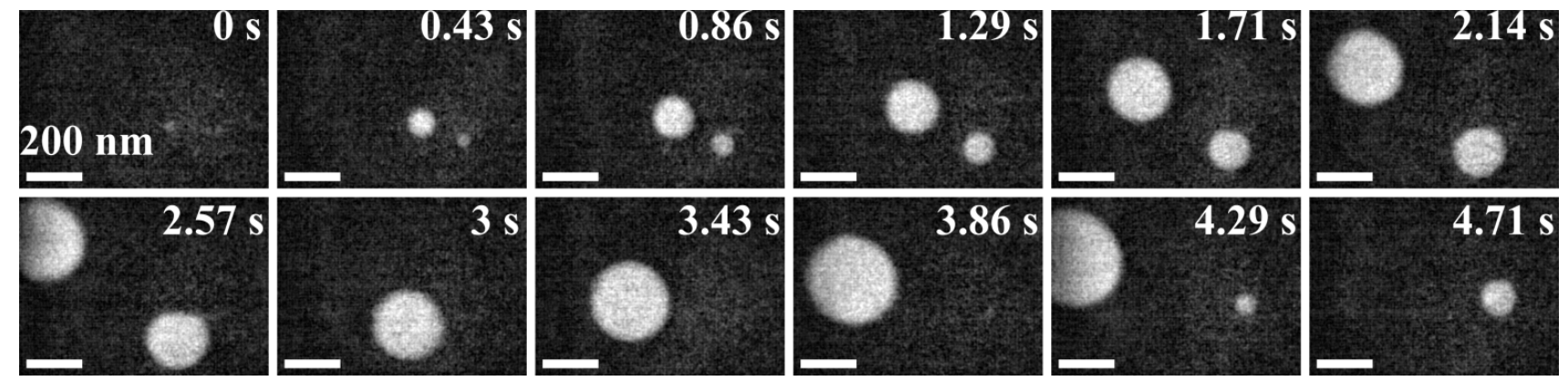

Figure 1: Frames from in situ TEM video of bubble nucleation and growth. $300 \mathrm{keV}$ electrons, beam current $I<1 \mathrm{nA}$, and beam radius $\sim 2 \mu \mathrm{m}$. Two bubbles nucleate on the membrane, grow, and detach. Another bubble nucleates at the same spot and the process repeats.

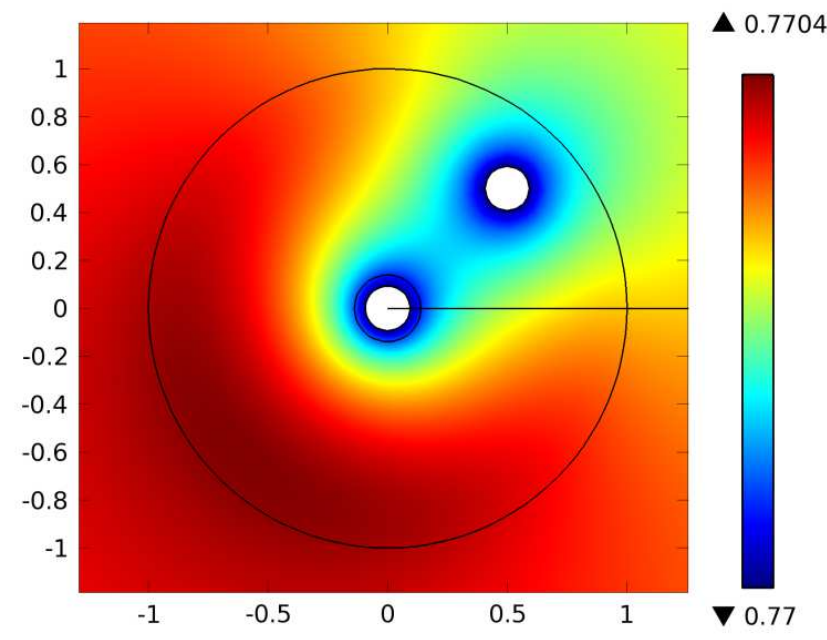

Figure 2: Modeling $\mathrm{H}_{2}$ concentration in the liquid cell. $300 \mathrm{keV}$ electrons with beam radius of $2 \mu \mathrm{m}$ (black circle of dimensionless radius 1), and beam current of $0.3 \mathrm{nA}$. The concentration distribution of $\mathrm{H}_{2}$ (in $\mathrm{mM}$ ) is shown when two bubbles (white voids) with radius $185 \mathrm{~nm}$ are present. $C_{\text {sat }}(100 \mathrm{kPa})=0.77 \mathrm{mM}$ is specified at the boundaries of the bubbles. 